Table 1 Shiwei-Wendan decoction on the effects of 5-HT syndrome about threwing the head times in mice

Group
Model group
Haloperidol group
Shiwei-
Wendandecoction

Pre-dose

$31.8571 \pm 2.6726$

$32.2857 \pm 2.9277$

$32.7143 \pm 1.7995$

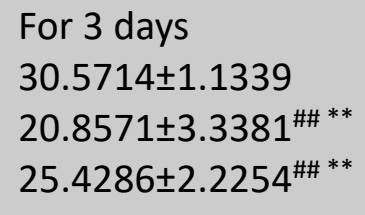

For10 days

$29.5714 \pm 1.5119$

$13.4286 \pm 2.8200^{\# \# \Delta \Delta^{* *}}$

$20.7143 \pm 3.3523^{\# \# \Delta^{* *}}$

Note : within the group comparison:compared with before the dosing, \# \# $<0.01$; Compared with dosing day 3 , $\Delta \Delta p<0.01 \Delta p<0.05$.Comparison between groups : compared with model group, ${ }^{*} * \mathrm{P}<0.01$; Compared with haloperidol group, $\mathbf{\Delta} P<0.05, \boldsymbol{\Delta} \Delta \mathrm{P}<0.01$.

Show that Shiwei-Wendan decoction can obviously reduce the schizophrenia 5 - HT syndrome model mice of shaking head times caused by 5-HTP and improve the 5 - HT sample symptoms of schizophrenia.

4 Discussion This study applies the model to explore the role of Shiwei-Wendan decoction related to 5-HT receptor. Shiwei-Wendan decoction has the phlegm and go qi、tonify, nerves on volunteers, it used to treat on phlegm and qi stagnation, deficiency of qi of heart and gallbladder,liver and spleen discord,to cause through agitated, insomnia, paranoid, easily frightened the madness of disease.The results show that Shiwei-Wendan decoction can obviously reduce the schizophrenia 5-HT syndrome model mice shaking head times caused by5-HTP.Improve the 5-HT sample symptoms of schizophrenia.Its for $5-\mathrm{HT}$ receptor antagonism have a certain extent effect,it provide a basis for Shiwei-Wendan decoction' clinical treatment of schizophrenia, It's worth to further research.

DOI 10.22448/AMJ.2017.3.21-21

\title{
THE CLINICAL RESEARCH OF ACUPUNCTURE ON IMPROVING COGNITIVE FUNCTION OF PATIENTS WITH VASCULAR DEMENTIA
}

\section{Chen Jing Wang Linjing Hou Xinlei An Wei}

the Acupuncture Five Ward of the Second Affiliated Hospital of Heilongjiang University of Chinese Medicine

Email:chenjing108106@163.com Postal addresses: the Acupuncture Five Ward of the Second Affiliated Hospital of Heilongjiang University of Chinese Medicine

Abstract Objective: Studying the improvement of acupuncture on cognitive function of patients with vascular dementia (VD), to provide reliable evidence of clinical acupuncture.

Method: 60 patients with VD were randomly divided into the acupuncture group, 30 cases and the drug control group, 30 cases. The acupuncture group:The main point: Baihui, Shenting, Meichong, Fengchi, Shuaigu, Taixi, Xuanzhong, Shenmen. The acupuncture methods: With general skin disinfection, take 30 1.0-1. 5 inch needle, the needle body pierces into Baihui with 15 degrees between the skin (to the left and right sides of Si Shencong), Shenting (back through the thorns on the star points), to the subgaleal, then twist pin (frequency about 200 times / min) $2 \mathrm{~min}$, needle retention $40 \mathrm{~min}$. The direction of the Fengchi point to the tip of the nose piercing, other points also taking all 30-inch $1.0-2.0 \mathrm{~mm}$ needle piercing into, ping-ping up the onset underwent reducing method, needle retention $40 \mathrm{~min}$. Twice a day on weekdays, except Sunday, 2 weeks is a course of treatment, a total of 4 courses. The drug control group:Oraling Duxil (Duxil Servier pharmaceutical production), Each $40 \mathrm{mg}, 2$ times a day. Service 6 days a week, physical interest one day, 2 weeks to a course of treatment, a total of 4 courses. Take assessment of HDS-R scores before and after treatment. Evaluation standard: Markedly: HDS-R score increased 10 points or more; effective: HDS-R score increased by 1 to $9 \mathrm{pm}$; invalid: HDS-R score unchanged or decreased.

Results: the acupuncture group can improve the VD patients, HDS-R score, the total effective rate $86.67 \%$, compare with the drug control group , there were significant differences between the two groups $(P<0.05)$

Conclusion: Acupuncture can improve the VD patients, cognitive function and the quality of life.

Key words: Acupuncture; Vascular Dementia; Cognitive Function; Clinical Research

References

[1] Wang Weizhi rule of Neurology, [M] Beijing: People's Medical Publishing House, 2002.253

[2] Chen Qingtang Stroke rate in patients with neurological impairment standard [J]. Journal of Neurology .1996, 29 (6): 381

[3] Cheng Zhanpei The modified Hasegawa-style mini mental assessment scale [J]. Foreign Medical • Psychiatry volumes, 1993,20 (1): $40-42$

[4] Lin Hai. Huangxue Qi. Sun Sulun. Vascular dementia model rats of acetylcholine content and Jiannao side effects of its intervention [J]. Journal of Traditional Chinese Medicine, 200836 (5): 24 - 25.

[5] Huang Yong. Lai Xinsheng. Chen Jing, etc. before and after acupuncture Baihui patients with vascular dementia in different brain regions of the cerebral glucose metabolism [J], Chinese Journal of Clinical Rehabilitation, 2005,9 (36):68-70.

[6] Zhang Ping, Ni Lanzhi Fengchi, Fengfu sequelae of stroke research [J] Chinese Acupuncture since.1998. 12: 743 